\title{
STABILITY OF TYPICAL CONTINUOUS FUNCTIONS WITH RESPECT TO SOME PROPERTIES OF THEIR ITERATES
}

\author{
J. SMITAL AND K. NEUBRUNNOVÁ
}

\begin{abstract}
Let $I$ be a real compact interval, and let $C$ be the space of continuous functions $I \rightarrow I$ with the uniform metric. For $f \in C$ denote $\nu(f)=$ $\sup _{x \in I}\left(\lim \sup _{n \rightarrow x} f^{n}(x)-\liminf _{n \rightarrow x} f^{n}(x)\right)$, where $f^{n}$ is the $n$th iterate of $f$. Then for each positive $d$ there is an open set $C^{*}$ dense in $C$ such that the oscillation of $\nu$ at each point of $C^{*}$ is less than $d$. Consequently, $\nu$ is continuous in $C$ except of the points of a first Baire category set.
\end{abstract}

Let $I$ be a compact real interval, $C$ the metric space of continuous functions $I \rightarrow I$ with the uniform metric. For $f \in C$ let $\|f\|=\max \{f(x) ; x \in I\}$, and let $f^{n}$ denote the $n$th iterate of $f$. If $f$ has a cycle $x_{1} \mapsto x_{2} \mapsto \cdots \mapsto x_{m} \mapsto x_{1}$, where $x_{i} \neq x_{j}$ for $i \neq j, i, j=1, \ldots, m$, then the order of this cycle is $m$ while the width of this cycle is $\max \left(x_{i}-x_{j}\right)$. Let $\lambda(f)$ be the l.u.b. of the widths of all cycles of $f$, and put

$$
\nu(f)=\sup _{x \in I}\left(\limsup _{n \rightarrow \infty} f^{n}(x)-\liminf _{n \rightarrow \infty} f^{n}(x)\right) .
$$

Clearly $0 \leqslant \lambda(f) \leqslant \nu(f)$ for each $f \in C$, and $\nu(f)=0$ iff $f$ has no cycles (cf. [4]). The function $\nu$ can be sometimes used as a "measure of chaos". Namely, if $f \in C$ has no cycles, then each neighbourhood of $f$ contains a chaotic function (cf. [2]). But if additionally the set of fixed points of $f$ contains no interval, then for each $g$ sufficiently near to $f, \nu(g)$ is small (although it can be positive) (cf. [5]).

L. Block [1] recently has shown that continuous functions are stable with respect to the order of their cycles: if $f \in C$ has a cycle of order $m$, and if $n$ is greater than $m$ in the Šarkovskii ordering (cf. [3 or 6]), then each $g \in C$ sufficiently near to $f$ has some $n$-cycle. However, Block's result gives no information on the width of the corresponding cycles. As we show (see Theorem 1) the width of the cycles of functions $g$ from a certain neighbourhood of $f$ can be essentially smaller then the width of cycles of $f$, i.e. $\lambda(g) \ll \lambda(f)$, and in application the cycles of $g$ then cannot be distinguished from the noise. The main aim of this paper is to show that such a type of behaviour is untypical. We begin with the following example.

THEOREM 1. Given a $\delta>0$ there is a continuous $f:[0,1] \rightarrow[0,1]$ with the following properties: $\lambda(f)=1$ and for each $\varepsilon>0$ there is some continuous $g:[0,1] \rightarrow[0,1]$ such that $\|f-g\|<\varepsilon$ and $\nu(g)<\delta$.

Received by the editors August 16, 1982 and, in revised form, December 16, 1982.

1980 Mathematics Subject Classification. Primary 54H20; Secondary 26A18.

c) 1984 American Mathematical Society $0002-9939 / 84 \$ 1.00+\$ .25$ per page 
Proof. Let $I_{1}, I_{2}, \ldots, I_{2 n+1}$ be pairwise disjoint closed subintervals of $[0,1]$ with the natural ordering

$$
I_{2 n+1}<I_{2 n-1}<\cdots<I_{1}<I_{2}<I_{4}<\cdots<I_{2 n}
$$

(i.e. $x<y$ for each $x \in I_{3}$ and $y \in I_{1}$, etc.). Put $I_{i}=\left[x_{i}, y_{i}\right]$ for each $i$, and assume $x_{2 n+1}=0, y_{2 n}=1$. Define a function $f:[0,1] \rightarrow[0,1]$ in the following way: $f\left(I_{i}\right)=$ $I_{i+1}$ if $i \neq 2 n+1$ and $f\left(I_{2 n+1}\right)=I_{1}$. Moreover, $f$ is linear and decreasing on each $I_{i}$ where $i \neq 2 n+1$, and linear and increasing on $I_{2 n+1}$. Finally, extend $f$ continuously onto the whole $[0,1]$.

It is easy to verify that $f: x_{1} \mapsto y_{2} \mapsto x_{3} \mapsto y_{4} \mapsto \cdots \mapsto y_{2 n} \mapsto x_{2 n+1} \mapsto x_{1}$, i.e. $x_{1}$ generates a cycle of order $(2 n+1)$ and of the width 1 . Similarly $y_{1}$ is a point of a $(2 n+1)$-cycle, and from the linearity of $f$ on each $I_{i}$ we have $f^{2 n+1}$ is the identity mapping on $I_{1}$, and consequently, on each $I_{i}$. Thus each point of $I_{1}$ is a cyclic point of order $2 n+1$.

Now define a continuous $g:[0,1] \rightarrow[0,1]$ such that $g(x)=f(x)$ for $x \in I_{2 n+1}$, $g\left(x_{2 n+1}\right)=x_{1}+\varepsilon, g\left(y_{2 n+1}\right)=y_{1}$, and $g$ is linear on $I_{2 n+1}$. Without loss of generality we may assume that $x_{1}+\varepsilon<y_{1}$.

Clearly for each $x \in I_{2 n+1}$, except of $x=y_{2 n+1}$, we have $f(x)<g(x)=f(y)$ for some $y \in I_{2 n+1}, y>x$. Hence

$$
g^{2 n+1}(x)=f^{2 n+1}(y)=y>x .
$$

Thus $g$ has in $I_{2 n+1}$ exactly one cyclic point of order $>1$, namely the point $y_{2 n+1}$.

To finish the proof note that when the length of $I_{2 n+1}$ is greater than $1-\delta$, then we have $\lambda(g)<\delta$. Moreover, by (1) the cycle of $g$ generated by $y_{2 n+1}$ attracts all points of $I_{2 n+1}$, and this implies $\nu(g)<\delta$. Q.E.D.

REMARK. The theorem holds when $2 n+1$ is replaced by any $m>1$. Also Theorem 1 shows that neither $\nu$ nor $\lambda$ is lower semicontinuous. An example exhibiting that these functions are not upper semicontinuous can be found in [5].

In the proof of our main result the following lemmas are useful.

Lemma 1. Let $f \in C$ with $\nu(f)>d$. Then for each $\varepsilon>0$ there is some $g \in C$ such that $\|f-g\|<\varepsilon$ and $g$ has a cycle of the width greater than $d$.

Proof. Choose $x \in I$ such that

$$
\limsup _{n \rightarrow \infty} f^{n}(x)-\liminf _{n \rightarrow \infty} f^{n}(x)>d .
$$

For simplicity denote $f^{n}(x)=x_{n}$. Without loss of generality we may assume that the sequence $\left\{x_{n}\right\}$ is not periodic. Let $\delta>0$ be such that $|f(u)-f(v)|<\varepsilon$ whenever $|u-v|<\delta$ for $u, v \in I$. By (2) there are indexes $n(1)<n(2)<n(3)$ such that

$$
\left|x_{n(1)}-x_{n(3)}\right|<\delta
$$

and

$$
\left|x_{n(2)}-x_{n(3)}\right|>d \text {. }
$$

Now let $g\left(x_{n}\right)=f\left(x_{n}\right)$ for $n=n(1)+1, \ldots, n(3)-1, g\left(x_{n(3)}\right)=f\left(x_{n(1)}\right)$ and let $g$ be continuous in $I$. Moreover, by (3), $\left|g\left(x_{n(3)}\right)-f\left(x_{n(3)}\right)\right|<\varepsilon$ hence we can choose $g$ 
such that $\|f-g\|<\varepsilon$. It is easy to verify that $g$ has a cycle $x_{n(1)+1} \mapsto x_{n(1)+2} \mapsto$ $\cdots \mapsto x_{n(3)} \mapsto x_{n(1)+1}$ of order $k=n(3)-n(1)$. By (4) the width of this cycle is greater than $d$. Q.E.D.

Lemma 2. Assume that $f \in C$ and that $\lambda(f)>d$. Then for each $\varepsilon>0$ there is some $g \in C$ with the following properties: $\|f-g\|<\varepsilon$ and for each $h \in C$ from a sufficiently small neighbourhood of $g$ we have $\lambda(h)>d$.

Proof. Choose a cycle $x_{1} \mapsto x_{2} \mapsto \cdots \mapsto x_{n} \mapsto x_{1}$ of the function $f$ of the width $d_{1}>d$. First assume that one of the points $x_{i}$, say $x_{1}$, is an interior point of $I$. Choose also $\delta$ with $0<\delta<\left(d_{1}-d\right) / 2$ such that for every $u, v \in I$,

$$
|u-v|<\delta \text { implies }|f(u)-f(v)|<\varepsilon .
$$

Moreover, let $U_{1}$ be an open interval containing $x_{1}$ and denote $U_{i}=f^{i-1}\left(U_{1}\right)$, $i=2, \ldots, n$. Without loss of generality we may assume that $U_{1}$ is so small that the sets $U_{i}$ are pairwise disjoint, and that the length of $U_{1}$ is less than $\delta$. Now choose $u, v \in U_{1}$ such that $u<x_{1}<v$. Define $g$ by $g(y)=f(y)$ for $y \notin U_{1}, g(u)=g(v)=$ $x_{2}$, and extend $g$ continuously to a function $I \rightarrow I$. By (5) $g$ can be chosen such that $\|f-g\|<\varepsilon$. We have

$$
g^{n}(u)=x_{1}>u \text { and } g^{n}(v)=x_{1}<v .
$$

If $h \in C$ is near to $g$, then $h^{i}((u, v)) \subset U_{i+1}, i=1, \ldots, n-1$, and by $(6), h^{n}(u)>u$, $h^{n}(v)<v$. Hence $h$ has a periodic point $\xi \in(u, v)$. The order of $\xi$ must be $n$ since $h^{i}(\xi) \in U_{i+1}, i=1, \ldots, n-1$. Also it is easy to see that the width of this cycle is greater than $d_{1}-2 \delta>d$.

It remains to consider the case when $n=2$ and $x_{1}, x_{2}$ are the endpoints of $I$. Assume that $x_{1}<x_{2}$ and define $g$ by $g(x)=x_{2}$ for $x \in\left[x_{1}, x_{1}+\mu\right]$ and $g(x)=x_{1}$ for $x \in\left[x_{2}-\mu, x_{2}\right]$, where $\mu>0$ is small. Moreover, let $g(x)=f(x)$ for $x \in\left[x_{1}+\right.$ $2 \mu, x_{2}-2 \mu$ ], and let $g$ be continuous in $I$. Clearly for $\mu$ sufficiently small $g$ can be chosen such that $\|f-g\|<\varepsilon$. Also assume that $x_{2}-x_{1}-2 \mu>d$. Now let $h \in C$ with $\|h-g\|<\mu / 2$. Then $h^{2}\left(x_{1}+\mu\right)=x_{1}<x_{1}+\mu$. On the other hand, since $h$ : $I \rightarrow I$, we have $h\left(x_{1}\right) \geqslant x_{1}$. Thus for some $\xi \in\left[x_{1}, x_{1}+\mu\right), h^{2}(\xi)=\xi$ and clearly $h(\xi) \neq \xi$. It is easy to see that $|h(\xi)-\xi|>x_{2}-x_{1}-2 \mu>d$, i.e. $\lambda(h)>d$, and the lemma is proved.

In the following the oscillation $\omega_{\nu}(f)$ of $\nu$ at $f \in C$ is defined by

$$
\omega_{\nu}(f)=\lim \sup |\nu(g)-\nu(f)| \quad \text { for }\|g-f\| \rightarrow 0, g \in C,
$$

and similarly we define $\omega_{\lambda}(f)$. Now we are able to give the main result.

THEOREM 2. Let $\delta>0$. Then there is a subset $C_{\delta}$ of $C$, which is nowhere dense in $C$ and such that the oscillation both of $\nu$ and $\lambda$ at each $f \in C \backslash C_{\delta}$ is less than $\delta$.

In other words, when $\delta$ is small, both functions $\nu$ and $\lambda$ are continuous in the points of the set $C \backslash C_{\delta}$ up to small perturbations.

COROLlaRY. There is a first Baire category set $K \subset C$ such that both $\nu$ and $\lambda$ are continuous in the points of the set $C \backslash K$. 
Proof. Put $K=\cup_{n=1}^{\infty} c_{1 / n}$.

Proof of Theorem 1. Fix some positive integer $n$. Let $A_{i}=\{f \in C ; \nu(f)>i / n\}$ for $i=1, \ldots, n$, and put $A_{0}=C, A_{n+1}=\varnothing$. Let $B_{i}$ be the boundary of $A_{i}$, i.e. $B_{i}=\operatorname{Clos} A_{i} \cap \operatorname{Clos}\left(C \backslash A_{i}\right)$, where Clos is the closure operator in $C$. Then each $B_{i}$ is closed and nowhere dense in $C$. To see the second property, let $G$ be an open set. It suffices to consider the case $G \cap A_{i} \neq \varnothing$, where $i \neq 0$. By Lemma 1 there is some $f \in G$ with $\lambda(f)>i / n$, and by Lemma 2 there is a nonempty open subset $H \subset G$ such that $\lambda(h)>i / n$, and hence $\nu(h)>i / n$ for each $h \in H$. Thus $H \cap B_{i}=\varnothing$.

Now put $B=B_{1} \cup \cdots \cup B_{n}$. Let $f \in C \backslash B$ and let $j$ be the greatest index with $f \in A_{j}$. Then $f$ is an interior point of $A_{j}$ and $f$ does not belong to the boundary of $A_{j+1} \subset A_{j}$; hence $f \in A_{j} \backslash \operatorname{Clos} A_{j+1}$. Now it is easy to see that $\omega_{\nu}(f) \leqslant 1 / n$.

Similarly we can choose a nowhere dense set $D \subset C$ such that $\omega_{\lambda}(f) \leqslant 1 / n$ for each $f \in C \backslash D$. To finish the proof take $n$ such that $1 / n<\delta$ and put $C_{\delta}=B \cup D$.

REMARK. There is an open problem, whether for some $f, \lambda(f)<\nu(f)$. We conjecture that the answer is positive, but we have no example of such a function.

\section{REFERENCES}

1. L. Block, Stability of periodic orbits in the theorem of Šarkovskii, Proc. Amer. Math. Soc. 81 (1981), 333-336.

2. P. E. Kloeden, Chaotic difference equations are dense, Bull. Austral. Math. Soc. 15 (1976), 371-379.

3. A. N. Šarkovskii, Coexistence of cycles of a continuous transformation of a line into itself, Ukrain. Mat. Ž. 16 (1964), no. 1, 61-71. (Russian)

4. , On cycles and the structure of a continous transformation, Ukrain. Mat. Ž. 17 (1965), no. 3 , $104-111$.

5. J. Smital and K. Smitalová, Structural stability of non-chaotic difference equations, J. Math. Anal. Appl. 89 (1982).

6. P. Štefan, A theorem of Šarkovskii on the existence of periodic orbits of continuous endomorphisms of the real line, Comm. Math. Phys. 54 (1977), 237-248.

Department of Mathematics, Komensky University, 84215 Bratislava, Czechoslovakia 